\title{
Squatting, a posture test for studying cardiovascular autonomic neuropathy in diabetes
}

\author{
J.C. Philips, M. Marchand, A.J. Scheen
}

Division of Diabetes, Nutrition and Metabolic Disorders, Department of Medicine, CHU Sart-Tilman, 4000 Liège, Belgium

\begin{abstract}
Cardiovascular autonomic neuropathy (CAN) is a frequent complication of diabetes mellitus, which is associated with increased morbidity and mortality. It involves both the parasympathetic and sympathetic nervous systems, and may be diagnosed by classical dynamic tests with measurements of heart rate (HR) and/or arterial blood pressure (BP). An original squattest (1-min standing, 1-min squatting, 1-min standing) was used with continuous monitoring of HR and BP, using a Finapres ${ }^{\circledR}$ device. This active test imposes greater postural stress than the passive head-up tilt test, and provokes large changes in BP and HR that can be analyzed to derive indices of CAN. In healthy subjects, squatting is associated with BP increases and HR decreases (abolished by atropine: SqTv index), whereas the squat-stand transition is accompanied by a deep but transient drop in BP associated with sympathetic-driven tachycardia (abolished by propranolol: SqTs index). In diabetic patients with CAN, BP increases are accentuated during squatting whereas reflex bradycardia is reduced. When standing from squatting position, the fall in BP tends to be more pronounced and orthostatic hypotension more prolonged, while reflex tachycardia is markedly dampened. The baroreflex gain, similar to that calculated during pharmacological testing with vasodilator/vasopressor agents, can be derived by plotting pulse intervals (R-R) against systolic BP levels during the biphasic response following the squat-stand transition. The slope, which represents baroreflex sensitivity, is significantly reduced in patients with CAN. This discriminatory index allows study of the natural history of CAN in a large cohort of diabetic patients.
\end{abstract}

Keywords: Baroreflex sensitivity; Cardiovascular autonomic neuropathy; Finapres ; Squatting; Diabetes; Heart rate; Orthostatic hypotension; Posture test; Review

\section{Résumé}

Test postural d' accroupissement pour étudier la neuropathie autonome cardiovasculaire diabétique.

La neuropathie autonome cardiaque (NAC) est une complication fréquente du diabète qui est associée à une augmentation de la morbidité et de la mortalité. Elle concerne à la fois les systèmes parasympathique et sympathique et peut être diagnostiquée classiquement par des tests dynamiques avec mesure des variations de la frequence cardiaque (FC) et de la pression artérielle (PA). Notre groupe a utilise un test postural original (1-min debout-1 -min accroupi ou « squatting »-1 -min debout) avec un monitorage continu de FC et PA avec un appareil Finapres ${ }^{\circledR}$. Ce test de redressement actif impose un stress postural plus prononcé que le test d'inclinaison passif sur une table basculante et provoque des variations amp les de PA et FC qui peuvent être analysées pour dériver des indices de NAC. Chez des sujets sains, le «squatting» entraîne une augmentation de PA et une diminution de FC (abolie par l'atropine : indice SqTv) alors que la transition accroupi-debout induit une chute importante mais transitoire de PA accompagnée d'une tachycardie d'origine sympathique (abolie par le propranolol : indice SqTs). Chez les patients diabétiques avec NAC, l'augmentation de PA durant le « squatting » est accentuée alors que la bradycardie réflexe est réduite. Lors du passage de la position accroupie à la position debout, la chute de PA tend à être plus marquee et surtout plus prolongée alors que la tachycardie réflexe est amputee. Un gain baroréflexe, similaire à celui calculé lors d'un test pharmacologique avec des agents vasodilatateurs/vasopresseurs, peut être dérivé de la pente de la relation linéaire entre les intervalles R-R et la PA systolique suivant la transition accroupi-debout. Cette pente, qui représente la sensibilité du baroréflexe, est significativement diminuée chez les patients avec NAC. Cet indice performant nous a permis d'étudier l'histoire naturelle de la NAC dans une large cohorte de patients diabétiques. .

Mots clés : Baroréflexe ; Diabète ; Finapres ${ }^{\circledR}$; Fréquence cardiaque ; Hypotension orthostatique ; Neuropathie autonome cardiovasculaire ; Pression artérielle ; Revue générale 


\section{Introduction}

Cardiovascular autonomic neuropathy (CAN) is a classical, albeit underestimated, complication of diabetes mellitus (DM) in both type 1 (T1DM) and type 2 (T2DM) categories [1,2]. The most recognized features associated with CAN are reduced heart rate (HR) variability [3] and postural hypotension [4]. CAN is responsible for an increased risk in mortality, as revealed by a meta-analysis published in 2003 [5], and confirmed by two recent studies of patients with T1DM [6] and T2DM [7]. Therefore, the early diagnosis of CAN is recommended in clinical practice [1].

Non-invasive continuous monitoring of HR and arterial blood pressure (BP), using devices such as Finapres ${ }^{\circledR}$ and Finometer ${ }^{\circledR}$, has greatly facilitated the study of haemodynamic changes during posture tests in clinical research and practice $[8,9]$. Various posture tests are classically used to assess haemodynamic adaptation and autonomic function $[10,11]$. The most commonly used tests are the lying-to-standing active manoeuvre in clinical practice, and the head-up $70^{\circ}$-tilt table test passive manoeuvre in clinical research [11]. Another active test, the so-called squat test, produces the strongest orthostatic stress [10, 12], more so than the head-up tilt [10, $13,14]$, and can therefore be used to assess autonomic dysfunction [15]. Indeed, careful analysis of BP and HR changes during the transitions from standing to squatting and, even more interesting, from squatting to standing, provides invaluable information [8]. The squat test can be used to assess autonomic dysfunction especially through calculation ofthe so-called baroreflex gain [12, 15]. Our research group [16], as with others [13, 17, 18], was particularly interested in applying this original potent posture test to assess diabetic CAN.

The aim of the present review was to analyze the information obtained by continuously measuring BP and HR changes during a squat test to derive indices of CAN in patients with T1DM and T2DM (Table 1).

\section{Guidelines for the detection of cardiovascular autonomic neuropathy (CAN)}

The CAN Subcommittee of Toronto Consensus Panel on Diabetic Neuropathy recently published updated guidelines that were especially focused on the diagnosis and usefulness of CAN testing [1]. Criteria for CAN diagnosis and staging are:

- one abnormal cardiovagal test to identify possible or early CAN;

- at least two abnormal cardiovagal tests to identify definite or confirmed CAN;

- the presence of orthostatic hypotension, in addition to HR test abnormalities, to identify severe or advanced CAN.

Progressive stages of CAN are associated with an increasingly poorer prognosis.

The same committee critically reviewed the limitations and strengths of the available diagnostic approaches for CAN, and emphasized the need to develop new tests for autonomic function. It was concluded that the most sensitive and specific approaches currently available to evaluate CAN in clinical research are:

- HR variability;

- baroreflex sensitivity;

- muscle sympathetic nerve activity;

- plasma catecholamines;

- heart sympathetic imaging.

It was also recommended that efforts be undertaken to develop new non-invasive and safe CAN tests to be used in clinical research with higher sensitivity and specificity for studying the pathophysiology of CAN, as well as new therapeutic approaches [11].

\section{Haemodynamic changes during squatting and the squat-stand transition}

Our experimental squat test protocol (1-min standing, 1-min squatting, 1-min standing) included two successive transitions: first, from standing to squatting; and, second, from squatting to standing. Each transition provokes specific haemodynamic changes that can be monitored by a Finapres device, as recently described in detail elsewhere (Fig. 1) [12].

The transition from standing to squatting elicits muscle pumping and increased venous return, which leads to prompt increases in cardiac output, systolic BP, and pulse pressure (PP), followed by reflex bradycardia [ 12, 19]. Bradycardia was shown to be significantly dampened by the administration of atropine in healthy volunteers, consistent with vagal activation [17], and was not observed in heart transplant recipients [20]. Forearm vascular resistance was markedly decreased in squatting position in healthy individuals, but only minimally in patients with heart transplants, despite similar BP increases [20].

The squat-stand transition provokes an immediate sizeable reduction in BP, reflecting an instantaneous fall in systemic vascular resistance due to vasodilatation in the leg muscles (an effect that can be minimized by lower- 
body skeletal muscle-tensing) [21,22]. In normal, healthy subjects, cardiac output increases over the first $5 \mathrm{~s}$ after standing from squatting position due to compression of the leg and abdominal vessels. Rapid (within 15-20 s) BP recovery implies baroreflex stimulation [8], which is associated with tachycardia and secondary peripheral vasoconstriction [12,15]. Propranolol can markedly attenuate reflex tachycardia in normal subjects, suggesting that sympathetic activation plays a key role in this abrupt HR rise [17].

Table 1 : Summary of haemodynamic changes, derived indices and abnormalities in the presence of cardiovascular autonomic neuropathy (CAN) during the three successive phases of the squat test (standingsquatting-standing).

\begin{tabular}{llll}
\hline Posture & Standing & Squatting & Standing \\
\hline $\begin{array}{l}\text { Haemodynamic } \\
\text { changes }\end{array}$ & Baseline & $\uparrow$ BP & $\downarrow$ BP (transient) \\
& & $\uparrow$ PP $\downarrow$ HR & $\uparrow$ HR (transient) \\
$\begin{array}{l}\text { Squatting-derived } \\
\text { indices }\end{array}$ & & SqTv & SqTs \\
Abnormalities in & $\uparrow$ HR (fixed & Dampened & Baroreflex gain \\
presence of CAN & tachycardia) & bradycardia & tachycardia \\
& & $\uparrow$ SqTv & $\downarrow$ SqTs \\
& & $\uparrow$ BP & $\downarrow$ Baroreflex gain \\
& & $\uparrow$ PP & Orthostatic \\
& & $\uparrow$ Pulsatile stress & hypotension, \\
& & & (accentuated, \\
& & & prolonged)
\end{tabular}

$\uparrow$ : increased; $\downarrow$ : decreased; BP: blood pressure; PP: pulse pressure; HR: heart rate; SqTv: vagal index, or ratio between baseline cardiac R-R interval in initial standing position and longest R-R interval in the first $15 \mathrm{~s}$ of squatting; SqTs: sympathetic index, or ratio between baseline cardiac R-R interval and shortest R-R interval in the first 10 to $20 \mathrm{~s}$ of standing after squatting; Pulsatile stress $=\mathrm{PP} \times \mathrm{HR}$.

\section{Detection of diabetic CAN during a squat test}

\subsection{Heart rate changes}

In healthy individuals during autonomic blockade, HR changes are mainly controlled by vagal tone (blocked by atropine) during squatting and sympathetic tone (blocked by propranolol) during standing $[17,18]$. Therefore, such HR responses may be useful for assessing the functional integrity of the parasympathetic and sympathetic nerves, respectively, in patients with DM. Marfella and colleagues [17] proposed calculating a so-called vagal index (SqTv, the ratio between the baseline cardiac R-R interval in the initial standing position and the longest $\mathrm{R}-\mathrm{R}$ interval in the first $15 \mathrm{~s}$ of squatting) and a so-called sympathetic index (SqTs, the ratio between the baseline cardiac R-R interval and the shortest R-R interval in the first 10-20 s of standing after squatting). These indices, based on HR reduction during squatting and reflex tachycardia during standing, are considered markers of CAN: a higher SqTv value indicates parasympathetic neuropathy; while a lower SqTs is an indicator of sympathetic neuropathy (Table 1)[17].

Both SqTv and SqTs indices were calculated in a large cohort of healthy subjects and diabetic patients (one-third T1DM, two-thirds T2DM), all of whom submitted to standardized squat-testing [17]. Normal ranges of SqT ratios for healthy subjects aged 20-74 years showed a statistically significant negative correlation with age. The SqTv was outside the $99 \%$ confidence interval (CI) in $42 \%$ of the diabetic patients $v s 1.3 \%$ of the control subjects; the corresponding figures for the SqTs were $40 \%$ and $0.8 \%$, respectively. Age and duration of diabetes had a negative influence on both SqTv and SqTs ratios. In the same study, SqT ratios were compared with other reflex tests currently used for the diagnosis of CAN, including deep-breathing, lying-to-standing, the Valsalva manoeuvre and BP changes after standing (orthostatic hypotension) [2,11]. The conclusion was that SqT ratios can discriminate between healthy subjects and diabetic patients to an equal or greater extent than other tests, but are better than other tests on their own at identifying mild autonomic involvement [17].

The same Italian group reported that more diabetic patients treated with tolrestat, an aldose-reductase inhibitor, for 52 weeks had normalized SqTv or SqTs indices than those in the placebo group [23]. In another study, the same researchers reported that these squatting ratios significantly deteriorated after acute hyper-glycaemia in T2DM patients $(\mathrm{P}<0.01)$ [24]. Such changes were prevented by glutathione and reversed by L-arginine, suggesting their possible interference with endogenous nitric-oxide (NO) availability. Similar findings had been previously reported in healthy volunteers subjected to acute elevations of plasma glucose concentrations (15 $\mathrm{mmol} / \mathrm{L}$, a level similar to that observed in poorly-controlled diabetic patients); in that study, hyper-glycaemia 
produced relevant systemic haemodynamic changes (increases in BP and HR) and altered baroreflex activity (also assessed during a squat test). These haemodynamic changes were completely reversed by simultaneous infusion of glutathione, a free-radical scavenger that lowers oxidative stress, thereby suggesting a role for a glutathione-sensitive, presumably free-radical-mediated pathway [25].

Fig. 1. Changes in systolic blood pressure (SBP) and heart rate (beats/min) during a squat test (1-min standing, 1-min squatting, 1-min standing) in: (left column) a representative healthy subject $(R-R$ E/I ratio $=1.38) ;($ middle column) a patient with type 1 diabetes mellitus $(T 1 D M)$ without cardiovascular autonomic neuropathy $(C A N)(R-R$ E/I ratio= 1.41$)$; and (right column) a patient with T1DM complicated by severe cardiovascular autonomic neuropathy $(R-R E / I$ ratio $=1.08)$. The lowermost row shows the mode of calculation of baroreflex gain $(B R G)$ in each subject by plotting the pulse intervals $(R-R)$ against the SBP following the transition phase from squatting to standing.

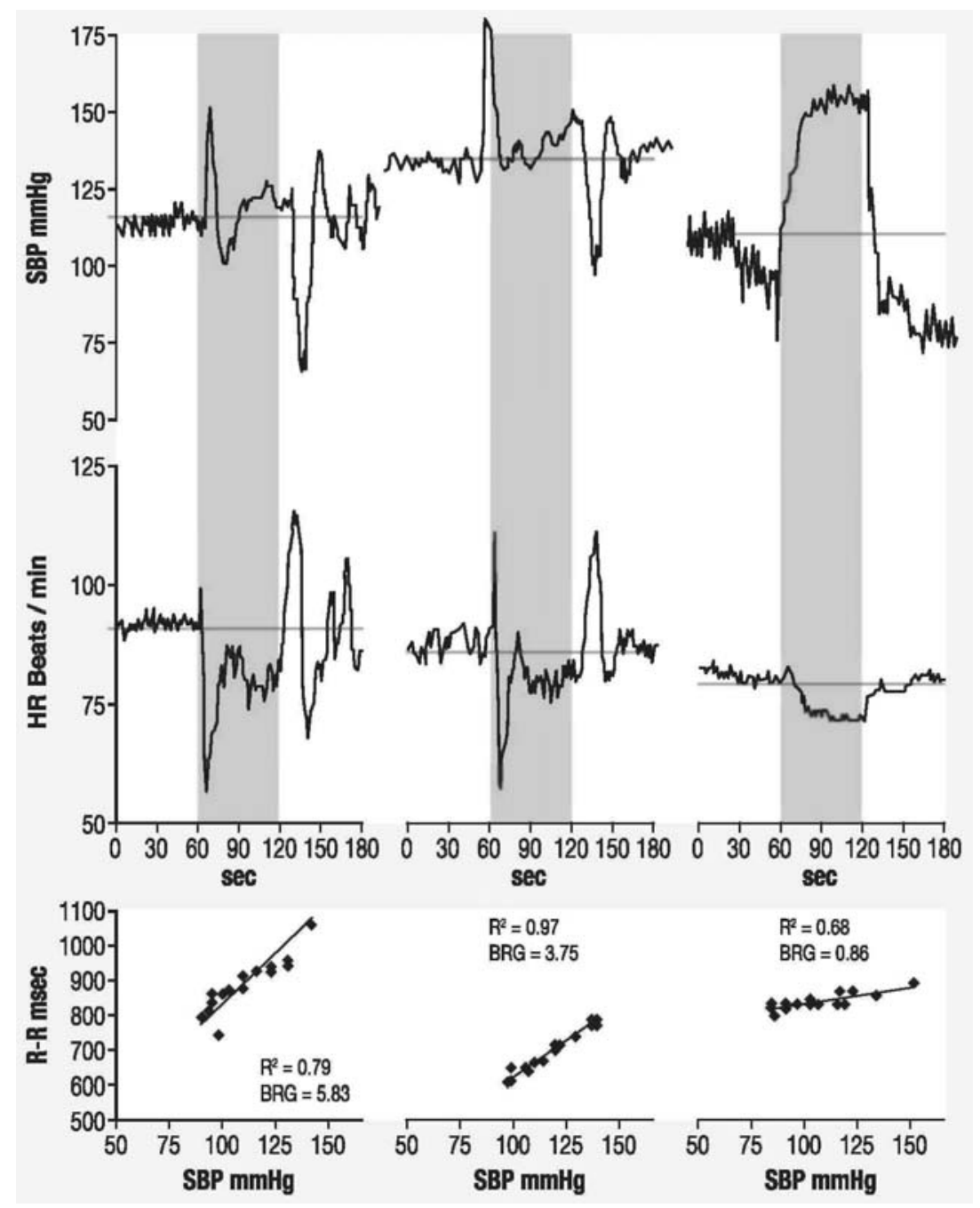

In a more recent study performed in Japanese T2DM patients, average changes in HR were calculated after completing the squatting (delta-HRSq) and standing (delta-HRSt) manoeuvre [18], and a positive correlation was noted between delta-HRSq and delta-HRSt $(r=0.86, P<0.0001)$. Furthermore, both indices significantly correlated with baroreflex sensitivity, as measured using the phenylephrine reference method (see below; $r=$ $0.66, P<0.0001$ and $\mathrm{r}=0.61, P<0.0001$, respectively). The authors concluded that these squatting-derived indices based on HR variations provide useful information for assessing CAN and for identifying diabetic patients at high risk of cardiovascular events.

In a cross-sectional controlled study, 159 patients with T1DM of various durations were evaluated during a 3min squat test [16]. CAN was assessed by the R-R interval expiration/inspiration (E/I) ratio during deep breathing, and by the SqTv and SqTs squatting-derived indices according to Marfella et al. [17]. While the R-R E/I ratio $(P<0.0001)$ progressively decreased with diabetes duration, the parasympathetic index SqTv significantly increased $(P<0.0001)$, whereas the sympathetic index SqTs only tended towards decrease $(P=$ 
0.12). In contrast, no such changes in CAN indices were observed in an age-matched non-diabetic population. Classically, parasympathetic dysfunction precedes sympathetic dysfunction in diabetic CAN [1,2]. In agreement with this observation, the SqTv index was markedly and significantly affected by diabetes duration, whereas changes in SqTs failed to reach the level of statistical significance in this T1DM population. However, in a larger database with a better balance according to age [26], SqTv significantly increased and SqTs significantly decreased across various age groups (from 20 to 60 years) in a T1DM population, as was the case for the R-R E/I ratio (Fig. 2). In a controlled age-, gender- and BMI (body mass index)-matched non-diabetic population, SqTv significantly decreased according to age, but not SqTs. Furthermore, it was confirmed that both SqTv and SqTs indices were significantly altered in diabetes on comparing middle-aged patients with long-duration T1DM and their age-matched healthy, non-diabetic controls [27].

In a recent study [28], haemodynamic changes during a squat test were compared in 20 T1DM patients with low R-R E/I ratios during a deep-breathing test (used as a marker of CAN) and 40 matched healthy subjects. Compared with the controls, the diabetic patients had lower R-R E/I ratios (1.05 vs 1.29; $P<0.00001)$, higher HR values throughout the posture test (90vs 82 beats. $\left.\mathrm{mnr}^{1} ; P=0.0252\right)$ and reduced reflex tachycardia following the transition from squatting to standing $\left(+18 v s+32\right.$ beats.mnr $\left.{ }^{1} ; P=0.0004\right)$, thereby significantly reducing SqTs (1.10 vs 1.22; $P=0.0001)$.

Fig. 2. Comparison of various indices (means $\pm S D$ ) of cardiovascular autonomic neuropathy (CAN) according to age (G1: 20-30 years, G2: 31-40 years; G3: $41-50$ years; G4: 51-60 years; $n=40$ in each group) in patients with type 1 diabetes (black histograms) vs age-, gender-and BMI (body mass index)-matched healthy subjects (white histograms): $R-R$ expiration/inspiration (E/I) ratio; vagal index (SqTv); sympathetic index (SqTs); and baroreflex gain (BRG). Statistical analysis used analysis of variance (Anova) for trends with age in each population (stars in boxes), and Student's unpaired t test for differences between diabetic patients and non-diabetic subjects in each age group (stars above each pair of histograms). Data are from the cohort reported in Philips et al. [26].
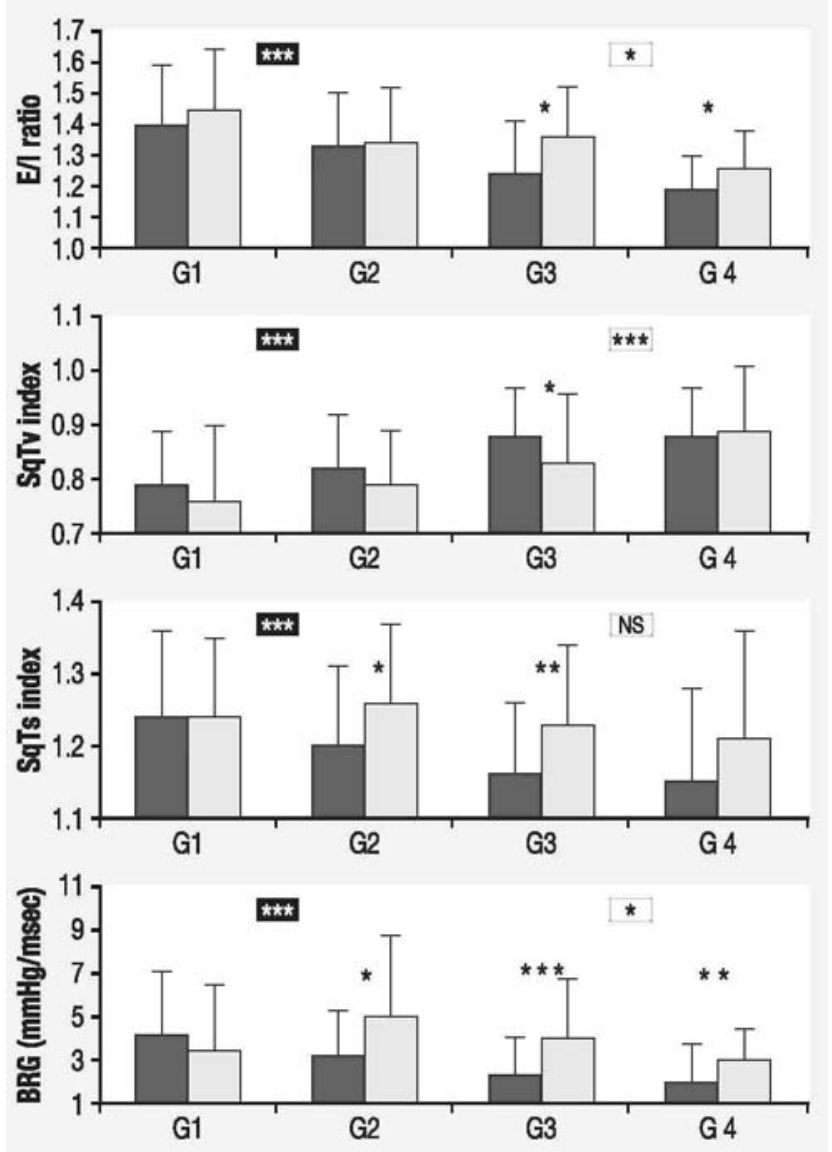

\subsection{Blood pressure changes}

The standing-squatting shift normally results in a transient first-phase BP burst followed by a secondary, more sustained, BP rise that, nevertheless, is still of somewhat small amplitude in healthy subjects (Fig. 1) [12]. It was reported in a large cohort of 159 T1DM patients that squatting was associated with a greater increase in BP and $\mathrm{PP}$ according to diabetes duration (especially after 20 years of disease duration) and age than in non-diabetic 
controls, a finding that was attributed to accelerated arterial stiffness resulting from chronic hyperglycaemia [16, 29]. In patients with T1DM and orthostatic hypotension (due to advanced CAN), a larger, sustained increase in $\mathrm{BP}$ was observed in the squatting position (Fig. 1), an observation in agreement with the use of squatting to counteract dizziness due to orthostatic hypotension in subjects with autonomic dysfunction [15]. However, whether the increase in BP and PP during squatting can exaggerate cardiovascular risk in certain conditions and/or some patients remains unknown.

The transition from squatting to standing results in an abrupt transient fall in BP that, in healthy subjects, is followed by a rapid return to baseline values according to a biphasic response (Fig. 1). In one detailed description, mean BP reached minimum after $7 \mathrm{~s}$ and, thereafter, increased to a new level, close to baseline level, within around $15 \mathrm{~s}$ [8].

A pilot study compared BP changes during three posture tests (lying-to-standing, sitting-to-standing and a squat test) in 10 healthy subjects, 10 T2DM patients without CAN and 10 T2DM patients with CAN assessed by the presence of a pathological R-R E/I ratio during a deep-breathing test [13]. A significantly greater BP drop was seen in T2DM patients with CAN compared with T2DM patients without CAN and the non-diabetic subjects $(P<0.001)$. Significant correlations were also observed between BP fall after squatting and either the R-R E/I ratio on deep breathing $(r=-0.77, P<0.001)$ or the duration of diabetes $(r=0.76, P<0.001)$. Thus, the intrinsic orthostatic load of the squat test, which is greater than those in conventional postural manoeuvres $[10,13,14]$, makes squatting a useful test for detecting early autonomic dysregulation leading to orthostatic hypotension $[13,15]$.

In a similar, more recent study [28], haemodynamic changes during an active orthostatic test were compared in 20 T1DM patients with low R-R E/I ratios during a deep-breathing test (used as a marker of CAN) and 40 matched healthy subjects. Compared with the controls, the diabetic patients did not exhibit any significantly greater initial BP drop but, instead, showed a delayed BP return to baseline after standing (33 vs 21s; $P=$ 0.0167); this abnormal response may be explained by dampened reflex tachycardia and presumably by reduced sympathetic-driven vasoconstriction.

\subsection{Baroreflex sensitivity}

Considered one of the most sensitive and specific approaches currently available to evaluate CAN in clinical research [11], the sensitivity of the arterial baroreflex (baroreflex gain, BRG) is routinely calculated from the relationship between HR increase and/or decrease responses (translated into respective R-R interval changes) and decreases and/or increases in arterial systolic BP [30,31]. The classical assessment of BRG uses a pharmacological approach, involving the infusion of angiotensin or phenylephrine to generate a vasopressor stimulus, associated with reflex bradycardia, and nitroprusside or amyl nitrite to induce vasodilatation and a fall in BP, associated with reflex tachycardia [32]. These pharmacological tools were designed to drastically perturb the system, thereby inducing rapid and large HR responses to facilitate the assessment of reflex gain. Indeed, baroreceptor responses are most apparent with rapidly changing pressures, rather than stationary or minimally changing BP levels during continuous monitoring under resting conditions [33]. It has also been reported that measurements of baroreflex sensitivity using non-invasive methods (Finapres) are highly correlated with measurements using intra-arterial invasive methods and can provide the equivalent prognostic information [34].

As previously reported by our group [16] and others [35, 36], and as recently described in detail [12, 16], the squat test can be used as an alternative dynamic procedure to assess BRG. During the transition from squatting to standing, there is an abrupt drop in BP associated with reflex tachycardia, and the initial changes are followed by a rapid return to the baseline values of both parameters (secondary BP increase and HR decrease; Fig. 1). Propranolol can markedly attenuate reflex tachycardia on standing from squatting $[17,18]$. The large biphasic mirrored changes in HR and systolic BP during a squat-stand test allow the calculation of BRG; indeed, such haemodynamic changes are almost identical to those seen with a vasodilator drug (hypotension with reflex tachycardia) and a vasoconstrictor agent (hypertension associated with bradycardia), although the haemodynamic changes occur more quickly in the squat test [18,30]. As previously described [12], BRG can be calculated by plotting the pulse intervals (R-R) against systolic BP following the transition phase from squatting to standing. The slope represents baroreflex sensitivity [36], and corresponds to changes in R-R pulse intervals per mm change in systolic BP (Fig. 1). Acceptable reproducibility of BRG measurement was also demonstrated in 70 (diabetic and non-diabetic) subjects who performed two squat-stand tests [12]. The BRG results in healthy subjects according to gender, BMI and age were also presented in a previous review [12]. 
Table 2: Comparison of baroreflex gain $(B R G)$ calculated during a squat-stand test in patients with type 1 diabetes (T1DM), according to various criteria, and patients with type 2 diabetes (T2DM).

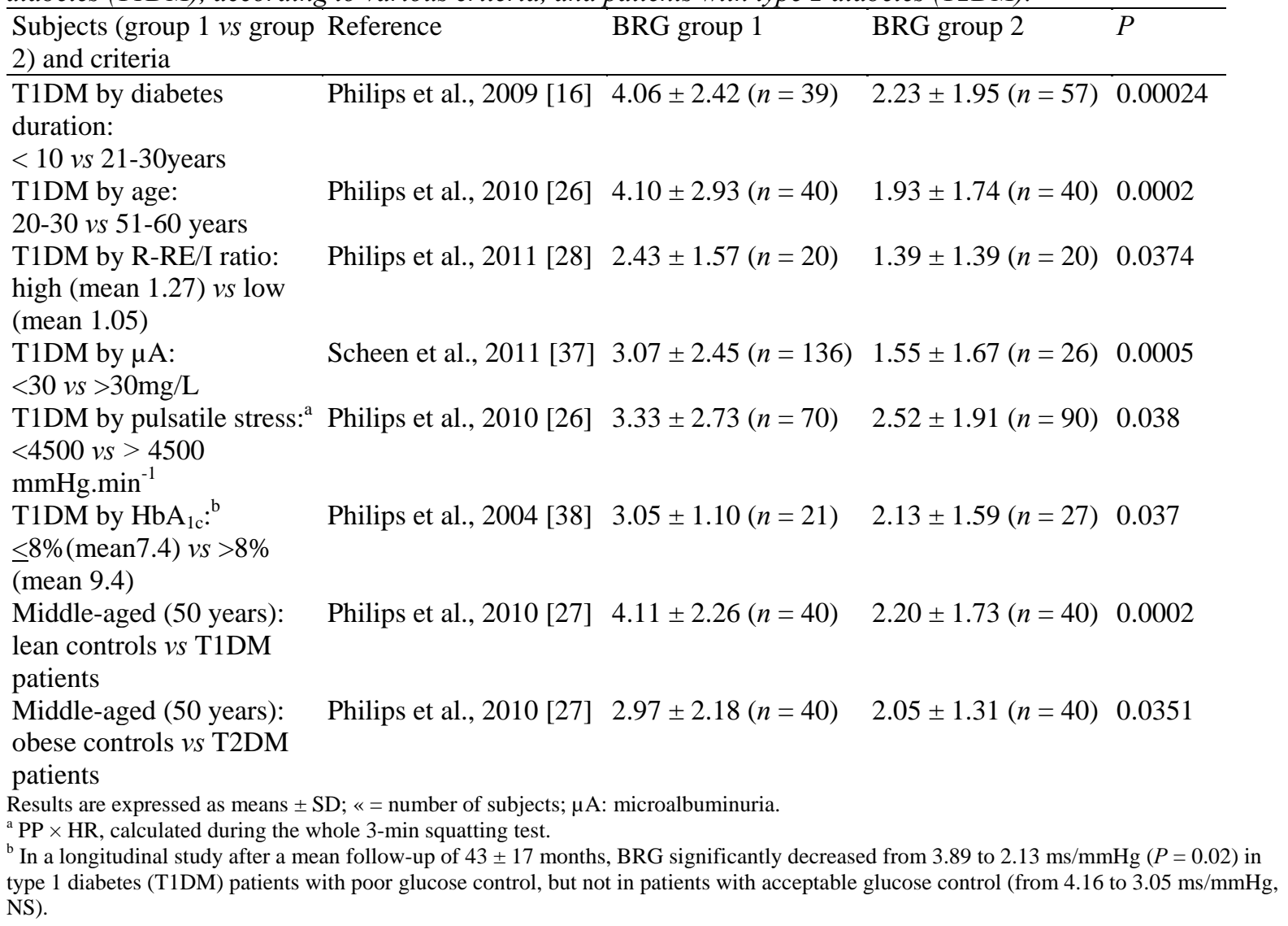

BRG measurements during a squat test have been used extensively by our group to assess CAN in diabetic individuals, mostly patients with T1DM. As summarized in Table 2, it was previously demonstrated that baroreflex gain is significantly reduced in T1DM patients with (a) longer disease duration [16], (b) greater age [26], (c) low R-R E/I ratios [28], (d) microalbuminuria ( $\mu \mathrm{A}$ ) [37], (e) increased pulsatile stress [26], (f) poor metabolic control [38] and (g) compared with age-matched middle-aged non-diabetic individuals (which was also the case for T2DM patients compared with weight- and age-matched healthy subjects) [27].

In addition, it was previously reported that BRG progressively decreased according to duration of disease in a population of patients with T1DM, whereas such areduction was not significant in a control non-diabetic population matched for age [16]. In a larger database [26], the progressive reduction in BRG according to age was more pronounced in T1DM patients compared with an age-, gender- and BMI-matched non-diabetic population (Fig. 2). Our results might suggest that BRG reduction occurs at an earlier stage than the R-R E/I ratio in the natural history of CAN in T1DM patients. These observations, obtained during a squat test, are in agreement with previous data reporting barore-ceptor cardiac reflex sensitivity measured during phase 4 of the Valsalva manoeuvre [39]. In that study, reductions in baroreflex sensitivity were detected in T1DM patients compared with controls (although standard tests of autonomic function revealed no significant differences). Furthermore, there were significant relationships between baroreflex impairment and the duration of diabetes $(P<0.001)$ and poor diabetes control $(P<0.05)$.

On comparing the relationship of two markers of CAN — the R-R E/I ratio measured during a deep-breathing test and BRG assessed during a squat test-with $\mu \mathrm{A}$ and pulsatile stress (the product of PP and HR) in 167 patients with T1DM [37], the patients with low BRG had an increased pulsatile stress index, especially in squatting position $(P<0.0001)$. Similarly, $\mu$ A was significantly higher in T1DM patients with low BRG $(P=$ $0.0001)$. Also, there was an inverse correlation between BRG and $\log \mu \mathrm{A}(r=-0.28, P=0.0006)$, but not between R-R E/I ratio and $\log \mu \mathrm{A}(r=0.09, P=0.12)$. Thus, the calculation of BRG during a squat-stand test in patients with T1DM allows for better detection of those patients with increased pulsatile stress, and even more so in patients with $\mu \mathrm{A}$, than the classical CAN index R-R E/I ratio. Thus, a decreased BRG measured during a squat test may be used to detect T1DM patients at high risk of cardiorenal complications compared with other current techniques [40]. 
In addition, to investigate the influence of blood glucose control on the progression of indices of CAN in patients with T1DM, 48 patients were subjected twice to a squat test over a time interval of $43 \pm 17$ months (range: 16-91 months) [38]. Overall blood glucose control was estimated by the mean value of several glycated haemoglobin $\left(\mathrm{HbA}_{1 \mathrm{c}}\right)$ measurements between the two tests, and patients were separated into two subgroups: HbA $\mathrm{H}_{1 \mathrm{c}}$ levels < $8 \%$ (in 21 patients; $7.42 \pm 0.60 \%$ ) $v s>8 \%$ (in 27 patients; $9.44 \pm 1.04 \%$ ). BRG tended to be negatively related $(P<0.10)$ to the duration of diabetes at the initial evaluation (19 \pm 10 years, range: $3-38$ years). Overall, this index of CAN decreased from $4.00 \pm 3.41$ to $2.53 \pm 1.46 \mathrm{~ms} / \mathrm{mmHg}$

$(P=0.008)$ during the 3.5-year period separating the two ortho-static tests. The BRG reduction was significant in patients with poor glucose control (from 3.89 to $2.13 \mathrm{~ms} / \mathrm{mmHg} ; P=0.02$ ), but not in patients with acceptable control (from 4.16 to $3.05 \mathrm{~ms} / \mathrm{mmHg}$; not significant, NS). While no significant differences were observed between the two groups at the initial evaluation, BRG was significantly lower at the second evaluation in the poorly-controlled vs better-controlled group $(P<0.05$; Table 2$)$. Significant negative correlations were also found between changes in BRG from test 1 to test 2 and mean $\mathrm{HbA}_{\mathrm{lc}}$ levels during the period between the two tests $(r=$ -0.304, $P<0.01$ ), and between BRG at the second evaluation and the corresponding averaged $\mathrm{HbA}_{1 \mathrm{c}}$ levels ( $r=-0.409, P<0.001$ ), thereby confirming previous observations from a cross-sectional study [39]. Thus, in patients with long-standing (almost 20 years) T1DM, a period of 3 to 4 years with poor glucose control is sufficient to significantly decrease BRG [38].

These longitudinal observations need to be confirmed in a larger cohort of patients with longer follow-up. Interestingly, in the Diabetes Control and Complications Trial (DCCT), T1DM patients (duration of disease $<15$ years) were randomly assigned to either conventional (mean $\mathrm{HbA}_{\mathrm{lc}}$ around 9\%) or intensive (mean $\mathrm{HbA}_{\mathrm{lc}}$ around $7 \%$ ) therapy and followed for up to 9 years. Baseline and biannual measurements of autonomic function (R-R E/I variation, Valsalva ratio and postural testing) were performed throughout the trial. In general, autonomic abnormalities increased; however, R-R variation was less frequently abnormal in the intensively treated and better-controlled T1DM patients (an almost 2\% difference in $\mathrm{HbA}_{\mathrm{lc}}$ level, as in our study) [41]. The Epidemiology of Diabetes Interventions and Complications (EDIC) study, a prospective observational follow-up of the DCCT cohort, reported persistent benefits with prior intensive therapy of CAN 13 to 14 years after the DCCT closeout. Prior DCCT intensive therapy (and, thus, better glucose control) reduced the risks of incident CAN by almost 30\% [42]. Similarly, in the Steno-2 trial, a target-driven, long-term, intensified intervention aimed at multiple risk factors in patients with T2DM (and also aiming to improve glucose control) reduced the risk of CAN by almost $60 \%$ after a mean follow-up of around 8 years [43]. These consistent results highlight the importance of good metabolic control in delaying the onset or slowing the progression of CAN in diabetic patients.

\section{Conclusion}

A recent consensus document critically reviewed the limitations and strengths of the available diagnostic approaches for CAN, and emphasized the need to develop new tests of autonomic function, although baroreflex sensitivity measurement is still considered to be among the most sensitive and specific approaches [11]. Our group used an original posture test, the squat test, to detect early signs of CAN in patients with DM. The substantial biphasic changes in systolic BP and HR arising during the initial squat-stand transition phase mimic those observed during pharmacological tests using vasodila-tor/vasopressor agents. By plotting the R-R intervals according to systolic BP changes following the squat-stand transitions, the calculated BRG may be used to assess cardiovagal baroreflex sensitivity and autonomic function. It was also demonstrated that a BRG reduction calculated during a squat test is more informative for detecting early CAN in patients with T1DM than a decreased R-R E/I ratio measured during a deep-breathing test, a classical CAN index widely used in clinical research, or other squatting-related indices derived only from HR changes. Although some individuals (such as the elderly and very obese) may find it difficult or even impossible to perform the squatting manoeuvre, in our experience, most patients with T1DM can be appropriately evaluated by this posture test.

\section{Disclosure of interest}

The authors declare that they have no conflicts of interest concerning this article.

\section{Acknowledgement}

The experimental work underlying this review was supported by an unrestricted research grant from Novo Nordisk Belgium. 


\section{References}

[1] Spallone V, Ziegler D, Freeman R, Bernardi L, Frontoni S, Pop-Busui R, et al. Cardiovascular autonomic neuropathy in diabetes: clinical impact, assessment, diagnosis, and management. Diabetes Metab Res Rev 2011; Epub 2011/06/23.

[2] Vinik AI, Ziegler D. Diabetic cardiovascular autonomic neuropathy. Circulation 2007;115:387-97.

[3] Risk M, Bril V, Broadbridge C, Cohen A. Heart rate variability measurement in diabetic neuropathy: review of methods. Diabetes Technol Ther 2001;3:63-76.

[4] Purewal TS, Watkins PJ. Postural hypotension in diabetic autonomic neuropathy: a review. DiabetMed 1995;12:192-200.

[5] Maser RE, Mitchell BD, Vinik AI, Freeman R. The association between cardiovascular autonomic neuropathy and mortality in individuals with diabetes: a meta-analysis. Diabetes Care 2003;26:1895-901.

[6] Soedamah-Muthu SS, Chaturvedi N, Witte DR, Stevens LK, Porta M, Fuller JH. Relationship between risk factors and mortality in type 1 diabetic patients in Europe: the Eurodiab prospective complications study (PCS). Diabetes Care 2008;31:1360-6.

[7] Pop-Busui R, Evans GW, Gerstein HC, Fonseca V, Fleg JL, Hoogwerf BJ, et al. Effects of cardiac autonomic dysfunction on mortality risk in the Action to control cardiovascular risk in diabetes (Accord) trial. Diabetes Care 2010;33:1578-84.

[8] Rossberg F, Penaz J. Initial cardiovascular response on change of posture from squatting to standing. Eur J Appl Physiol Occup Physiol 1988;57:93-7.

[9] Imholz BP, Settels JJ, van der Meiracker AH, Wesseling KH, Wieling W. Non-invasive continuous finger blood pressure measurement during orthostatic stress compared to intra-arterial pressure. Cardiovasc Res 1990;24:214-21.

[10] Scheen AJ, Juchmes J, Pochet T. Non invasive, beat-to-beat, investigation of the effects of posture on arterial blood pressure in diabetic neuropathy. Diab Metab 1990;16:382-4.

[11] Bernardi L, Spallone V, Stevens M, Hilsted J, Frontoni S, Pop-Busui R, et al. Investigation methods for cardiac autonomic function in human research studies. Diabetes Metab Res Rev 2011 ; Epub 2011/06/23.

[12] Scheen A, Philips J-C. Squatting test: a dynamic postural manoeuvre to study baroreflex sensitivity. Clin Aut Res 2011 ; Epub 2001/08/04.

[13] Marfella R, Salvatore T, Giugliano D, Di Maro G, Giunta R, Torella R, et al. Detection of early sympathetic cardiovascular neuropathy by squatting test in NIDDM. Diabetes Care 1994;17:149-51.

[14] Rickards CA, Newman DG. A comparative assessment of two techniques for investigating initial cardiovascular reflexes under acute orthostatic stress. Eur J Appl Physiol 2003;90:449-57.

[15] Philips JC, Scheen AJ. Squatting test: A posture to study and counteract cardiovascular abnormalities associated with autonomic dysfunction. Auton Neurosci 2011;162:3-9.

[16] Philips JC, Marchand M, Scheen AJ. Pulse pressure and cardiovascular autonomic neuropathy according to duration of type 1 diabetes. Diabetes Metab Res Rev 2009;25:442-51.

[17] Marfella R, Giugliano D, di Maro G, Acampora R, Giunta R, D'Onofrio F. The squatting test. A useful tool to assess both parasympathetic and sympathetic involvement of the cardiovascular autonomic neuropathy in diabetes. Diabetes 1994;43:607-12.

[18] Nakagawa M, Shinohara T, Anan F, Yufu K, Takahashi N, Okada N, et al. New squatting test indices are useful for assessing baroreflex sensitivity in diabetes mellitus. Diabet Med 2008;25:1309-15

[19] O'Donnell TV, Mc IM. The circulatory effects of squatting. Am Heart J 1962;64:347-56.

[20] Hanson P, Slane PR, Rueckert PA, Clark SV. Squatting revisited: comparison of haemodynamic responses in normal individuals and heart transplantation recipients. Br Heart J 1995;74:154-8.

[21] Krediet CT, Go-Schon IK, Kim YS, Linzer M, Van Lieshout JJ, Wieling W. Management of initial orthostatic hypotension: lower body muscle-tensing attenuates the transient arterial blood pressure decrease upon standing from squatting. Clin Sci (Lond) 2007;113:401-7.

[22] Krediet CT, Go-Schon IK, van Lieshout JJ, Wieling W. Optimizing squatting as a physical maneuver to prevent vasovagal syncope. Clin Auton Res 2008;18:179-86.

[23] Giugliano D, Acampora R, Marfella R, Di Maro G, De Rosa N, Misso L, et al. Tolrestat in the primary prevention of diabetic neuropathy. Diabetes Care 1995;18:536-41.

[24] Marfella R, Nappo F, De Angelis L, Paolisso G, Tagliamonte MR, Giugliano D. Haemodynamic effects of acute hyperglycemia in type 2 diabetic patients. Diabetes Care 2000;23:658-63.

[25] Marfella R, Verrazzo G, Acampora R, La Marca C, Giunta R, Lucarelli C, et al. Glutathione reverses systemic haemodynamic changes induced by acute hyperglycemia in healthy subjects. Am J Physiol 1995;268:E1167-73.

[26] Philips JC, Marchand M, Scheen AJ. Changes in pulse pressure, heart rate and the pulse pressure $\times$ heart rate product during squatting in type 1 diabetes according to age. Diab Med 2010;27:753-61.

[27] Philips JC, Marchand M, Scheen AJ. Pulsatile stress in middle-aged patients with type 1 or type 2 diabetes compared with non diabetic control subjects. Diabetes Care 2010;33:2424-9.

[28] Philips JC, Marchand M, Scheen AJ. Haemodynamic changes during a squat test, pulsatile stress and indices of cardiovascular autonomic neuropathy in patients with long-duration type 1 diabetes. Diabetes Metab 2011, doi:10.1016/j.diabet.2011.07.007.

[29] Philips JC, Marchand M, Scheen AJ. Squatting amplifies pulse pressure increase with disease duration in patients with type 1 diabetes. Diabetes Care2008;31:322-4 
[30] La Rovere MT, Pinna GD, Raczak G. Baroreflex sensitivity: measurement and clinical implications. Ann Noninvasive Electrocardiol 2008;13:191-207.

[31] Parati G, Di Rienzo M, Mancia G. How to measure baroreflex sensitivity: from the cardiovascular laboratory to daily life. J Hypertens 2000; 18:7-19.

[32] Rudas L, Crossman AA, Morillo CA, Halliwill JR, Tahvanainen KU, Kuusela TA, et al. Human sympathetic and vagal baroreflex responses to sequential nitroprusside and phenylephrine. Am J Physiol 1999;276:H1691-8.

[33] Lipman RD, Salisbury JK, Taylor JA. Spontaneous indices are inconsistent with arterial baroreflex gain. Hypertension 2003;42:481-7.

[34] Pinna GD, La Rovere MT, Maestri R, Mortara A, Bigger JT, Schwartz PJ. Comparison between invasive and noninvasive measurements of baroreflex sensitivity; implications for studies on risk stratification after a myocardial infarction. Eur Heart J 2000;21:1522-9.

[35] Nakagawa M, Takahashi N, Shinohara T, Taniguchi Y, Anan F, Yonemochi H, et al. Evaluation of autonomic function using posture change. Rinsho Byori 2006;54:838-43.

[36] Zhang R, Claassen JA, Shibata S, Kilic S, Martin-Cook K, Diaz-Arrastia R, et al. Arterial-cardiac baroreflex function: insights from repeated squat-stand maneuvers. Am J Physiol Regul Integr Comp Physiol 2009;297:R116-23.

[37] Scheen AJ, Marchand M, Philips JC. Decreased baroreflex gain more strongly predicts microalbuminuria and increased pulsatile stress than decreased RR E/I ratio in patients with type 1 diabetes. Diabetologia 2011;54 (Suppl 1):S464, 1149.

[38] Philips JC, Marchand M, Geronooz I, Scheen AJ. Influence of blood glucose control on the progression of cardiac autonomic neuropathy in Type 1 diabetes. Diabetologia 2004;47(Suppl 1):A368-9 [P1029].

[39] Weston PJ, James MA, Panerai R, McNally PG, Potter JF, Thurston H, et al. Abnormal baroreceptor-cardiac reflex sensitivity is not detected by conventional tests of autonomic function in patients with insulin-dependent diabetes mellitus. Clin Sci (Lond) 1996;91:59-64.

[40] Lefrandt JD, Smit AJ, Zeebregts CJ, Gans RO, Hoogenberg KH. Autonomic dysfunction in diabetes: a consequence of cardiovascular damage. Curr Diabetes Rev 2010;6:348-58.

[41] Group DR. The effect of intensive diabetes therapy on measures of autonomic nervous system function in the diabetes control and complications trial (DCCT). Diabetologia 1998;41:416-23.

[42] Pop-Busui R, Low PA, Waberski BH, Martin CL, Albers JW, Feld-man EL, et al. Effects of prior intensive insulin therapy on cardiac autonomic nervous system function in type 1 diabetes mellitus: the diabetes control and complications trial/epidemiology of diabetes interventions and complications study (DCCT/EDIC). Circulation 2009; 119: 2886-93.

[43] Gaede P, Vedel P, Larsen N, Jensen GV, Parving HH, Pedersen O. Mul-tifactorial intervention and cardiovascular disease in patients with type 2 diabetes. N Engl J Med 2003;348:383-93. 